\title{
Collaborative information seeking with Ant Colony Ranking in real-time
}

\author{
Tommaso Turchi ${ }^{1}$, Alessio Malizia ${ }^{1}$, Paola Castellucci ${ }^{2}$, and Kai Olsen ${ }^{3}$ \\ 1 Dept. of Computer Science, Brunel University, UK \\ \{name. surname\} @brunel.ac.uk \\ 2 Dept. of Arts and Humanities, University of Rome "La Sapienza", Italy \\ paola.castelluccieuniromal.it \\ 3 Molde College, Norway \\ kai.a.olsen@himolde.no
}

\begin{abstract}
In this paper we propose a new ranking algorithm based on Swarm Intelligence (SI), more specifically on Ant Colony Optimization (ACO), to improve search engines' performances and reduce the information overload by exploiting users' collective behaviour. We designed an online evaluation involving end users to test our algorithm in a real-world scenario dealing with informational queries. The development of a fully working prototype, based on the Wikipedia search engine, demonstrated promising preliminary results.
\end{abstract}

\section{Introduction}

If we go back to the bibliographical databases in the seventies, text retrieval was based on a Boolean search query on the keywords of a document; predetermined attributes such as date and file size were used to rank results based on their relevance. With faster computers and more direct access storage it became possible to search the full text of a document: relevance was then often determined by the number of occurrences of each search term in the document, for example in relation to document size. The first search engines on the Web used this approach.

However, search term occurrences are not a good indicator for relevance: for example, when trying to find information on "VW Golf" search engines directed the user to online advertisements for used cars as these had the keywords, combined with a high occurrence to length ratio. Google's PageRank algorithm saved the day: now the number of links to a site determined relevance, higher if the sites with the links also had a high ranking. Then the "VW Golf" query would direct the user to an official site for Volkswagen, which most users would find more relevant than the car-for-sale advertisements.

While the PageRank algorithm functions well and offers a notion of "relevance" that is shared by many users it has the disadvantage of being static. It may be enhanced by other data and other techniques, but is in principle based on the current structure of the Web. The algorithm will even freeze this picture, as the high ranking will make the important sites more important. New interesting sites may get a low PageRank value and be presented further down on the search engine results page, and may therefore be noticed only by the most persistent users. Thus, the algorithm that is used to determine relevance may be self-fulfilling. 
To make Web searching more dynamic we could try to exploit the experience of these "persistent" users: this could be done as easy as presenting an other-users-found-this list. For example, we have been looking for a small lightweight camera with GPS to take on hiking trips; our search terms are "compact camera GPS". After some effort, trying a site here, another there, we have found what we have been looking for. To indicate that this site is interesting we could use a feature recently introduced by Google, the Google $+1^{a}$ service. This is similar to Facebook's "like" button. However, it will also be possible to extract the "interesting site" information indirectly, for example by evaluating the time used on each site, how many links on the site that were explored, if the user printed anything from the site or if the "buy" button was clicked. The next user giving the same or similar search terms could then go directly to this site, following the "other-users-found-this" link.

In this paper we shall explore this concept and present a model that describes a trend towards new Web-searching paradigms, which are both social and dynamic. The idea is taken from biology, from the way ants forage for food: the image in itself refers also to the seminal work of Norbert Wiener, the pioneer both of Cybernetics and of the political approach to technology. Particularly in The Human Use of Human Beings [22], Wiener uses the figurative speech of ants as a distopic one. Ants are perceived as a meek colony instead of an unpredictable cluster of single ants, each with its own identity. Therefore, the most appreciated qualities of ants turn out as dangerous disadvantages: ants can be easily controlled by a totalitarian ideology just because of their well-organized and collaborative way of behaving.

Our approach to Web searching and ranking relies on the positive meaning of the metaphor of the ants. But, at the same time, it is important not to undervalue the possible risks in a collaborative approach. Any single "ant" (i.e. any user) has his own unique and value-added perspective and knowledge, and should then be helped to enhance his sense of awareness about his "singularity". Seen under contemporary eyes, "ants" can easily be exploited by a single-minded, market-oriented society, or by a too generalistic and massive search engine and ranking algorithm. Instead, it is time to break monopolistic ways to access the Web. The information need of any single user must be taken into consideration, interpreted, and fulfilled. To that aim, it is necessary to make use of different methodologies of analysis. A variety of distinctive research communities can cooperate like "virtual ants": interdisciplinary approaches will surely prove helpful to find new paths.

\section{Related Work}

\subsection{Search Engines}

Although more than half $(59 \%)$ of Internet users in the US use a Web search engine during a typical day ${ }^{\mathrm{b}}$, in general the users' degree of satisfaction with major search engines is - to the best of our knowledge - largely unsettled, and can only be investigated thanks to rather small studies conducted in experimental environments.

\footnotetext{
${ }^{a}$ http://www.google.com/+/learnmore/+1/

b http://www.pewinternet.org/2012/03/09/search-engine-use-2012/
} 
According to Silverstein [19] (1) a maximum of two queries is needed to solve users' information needs (67\%) and, usually, (2) users scan only the first page of results (58\%). However, Hawking et al. [10] state that $50 \%$ of proposed search results are irrelevant, thus there are complex informational needs most likely receiving irrelevant results.

Other studies pointed out the low degree of satisfaction with search engine: Fox et al. [7] devised a machine learning approach which employs users' actions (e.g. the time spent on a page, the scrolling usage, the page visits, etc.) concluding that users consider $28 \%$ of search sessions unsatisfactory and 30\% only partially satisfactory. Xu and Mease [25] have measured the average duration of a search session: typically, users end a session even without satisfying their informational need - after 3 minutes.

Summarizing, many users employ search engines to satisfy their informational needs and as a starting point of their Web browsing $[13,1,18]$; nevertheless, the search experience is far from being perfect, in fact a substantial amount of searches end up unsatisfied.

In this paper, we deal with the problem of improving search engines' performance by exploiting the actions performed by the users; the problem that we try to address is the information overload, i.e. the inability to take a decision due to the huge quantity of information obtained by the users. As a matter of fact, search engines are tools designed to help people solving their own informational needs and - as we have discussed previously - there is much room for improvements.

Persistent users must be taken in due consideration too. On the average, they are also highly specialized users, often with highly specific information needs [6]. The old and dismissed library catalogue, the traditional opac, or even the generalistic search engine are any use for them [26,3]. As has been appropriately underlined by the LIS research community, next generation catalog (NGC) is tremendously needed, and can cooperate with web-scale discovery service [12].

\subsection{Collaborative Filtering}

Large scale searching on the Web can be applied only after a careful qualitative and quantitative analysis of user's satisfaction [2,16]. Here the problem of information overload if faced from a personalization perspective, without exploiting users' collective behaviour: they focus on personalizing search engines' results rather than improving their performances. LIS research community shares the same critical approach [20]. Therefore, it must be held in due consideration, looking for a synergic cooperation [17].

The first system employing a real Collaborative Filtering was the Tapestry mail system at Xerox PARC, described by Goldberg et al. [9] as "people collaborate to help one another perform filtering by recording their reactions to documents they read", which is thus considered orthogonal to content based filtering used by all the previously summarized systems. Following this preliminary study, several systems applied Collaborative Filtering to face information overload, like GroupLens [?] or Ringo [?], always asking users' feedback on suggested resources. [11].

Rucker and Polanco[?] devised a different and simpler approach in their Siteseer system: it collects users' bookmarks and use them both to find similar users and recommend bookmarks to other users that are unaware of them. In a similar manner, the PHOAKX system [?] inspects Usenet groups in order to find post containing URLs and recommendations, ranking them according to the number of posts. 
In the movie recommendations domain, examples of collaborative filtering are MovieLens, which is a recommendation system based on GroupLens technology [?], and RECommendation Tree (RecTree), which is a method using a divide-and-conquer approach to improve correlation-based collaborative filtering and performing clustering on ratings from users [?]. Finally, one of the most famous recommendation systems nowadays is the Amazon.com Recommendation [?].

\subsection{Information Foraging on the Web}

One of the theories trying to explain users' behavior while searching for information in complex systems (e.g. the Web) is the Information Foraging [?,?]; it is inspired by the optimal foraging theory, and one of its key concepts is the so-called "information scent", i.e. the perception the user has of the cost, value and ease to access of a resource, given some available clues (e.g. link, snippet, tag, comments, etc.). Applying the information foraging theory to Web information seeking seems quite natural and many approaches have been developed to improve this process, which can be traced back to this theory [?,?].

Nevertheless, many studies have chosen to extend the information provided by hyperlinks suggesting to users the most promising paths to follow in order to achieve their goals, usually working within a single website [?,?].

ScentTrails [15] continuously allows users to supply keywords and enriches hyperlinks providing a path that achieves the goal described by them. Finally, Wu and Aberer's method [23] operates within a single website, enriching the information provided by hyperlinks with a technique inspired by the ant foraging behavior (i.e. heavily clicked links are recommended in favor of less visited links).

\subsection{Other approaches}

There are many other ranking mechanisms exploiting users' behaviour, but with a different goal than personalizing the searching/browsing experience. A significant number of contributions comes from a "critical" analysis of PageRank. This "post-monopolistic" approach is currently pushing users beyond Google's way of evaluation, and towards new kind of "metrics" [4].

Probably the first search engine to take into account users' behavior in its ranking computation was the no longer available DirectHit, devised by Gary Culliss in 1995 and later bought by Ask Jeeves, which combined it with the Teoma search engine; it employed a ranking formula composed by three main factors: (1) content based ranking, (2) Link Analysis Ranking (LAR), and (3) usage based ranking; the latter takes into account all those clicks issued by users on a result in relation to a specific query, besides their own time of access and the time spent on the page.

Baeza-Yates et al. [?] devised a ranking algorithm in which the relevance of each document is boosted in relation to previous users' preferences; their method includes a preliminary phase of clustering, when similar queries are grouped. Then, the URLs are extracted and ordered by the number of clicks.

Summarizing, it's important to notice how none of the techniques just mentioned can be used to adapt a search engine to users in real time; in fact, all of them need to 
be periodically retrained to adapt the search engine's responses according to the last recorded usage behavior.

\section{Ant Colony Ranking}

We summarised some techniques to improve search engines' performance, highlighting a few key concepts: (1) the relationship between users seeking information and the optimal foraging theory; (2) the need of a search engine to adapt itself to users' behavior; and (3) the need to perform such adaptation in real time.

Almost none of the aforementioned approaches take all those three aspects into account - especially the latter - and those that do ([?]) might also benefit from a deeper implementation of some of the Information Foraging's key concepts.

As stated by Wu and Aberer[23] and by Olston and Chi [15], a swarm-based approach - thus one employing some SI ideas - can beyond a doubt take into account all those three key factors, being nonetheless a much more elegant and simple method than all the others 'ad-hoc' ones.

Thus, we'll now introduce a simple ranking algorithm based on SI that can be used to improve search engine's performance, adapting themselves to users' behavior.

Each day ants leave the colony in search of food and building materials; they will exploit the surroundings in all directions in a somewhat random fashion. If an ant finds anything of interest, it will return to the colony depositing pheromone, a chemical substance that ants are able to detect. Thus they create trails to signal the path between the colony and the food. The quantity of pheromone deposited, which may depend on the quantity and quality of the food, will guide other ants to the food source. That is, other ants in the colony may now use the pheromone as trail markers to reach the food. These markers evaporate over time, so that uninteresting trails disappear. Shorter trails will get a higher level of pheromone, thus shorter trails will endure longer, inserting a notion of ontimization. This form of organization may be used to characterize social behavior on $t \bigcirc z b$.

S1 ivers to the emergence of "intelligent" behaviours from a group of simple and/or loosely organized agents. Ants are a typical example of SI and their use of stigmergic processes ${ }^{\mathrm{c}}$ inspired the famous family of $\mathrm{ACO}^{\mathrm{d}}$ algorithms.

As we have previously stated, we will adapt the strategies employed in food searching by ant colonies in the building of ranking algorithms employing users' behaviour: it's pretty intuitive to find a parallelism between the way ants forage for food and the way users employ search engines to satisfy their informational needs; yet the latters don't leave any trace at all, so they can't provide any clues to the next users with their same informational needs, and - as about $30-40 \%$ of queries issued to a search engine are already been submitted [24] - that's a pretty common scenario.

We now propose a simple algorithm that implements the model we just proposed; we called it NaïveRank. We will assume that interactions between users and search engine

\footnotetext{
${ }^{c}$ The term was introduced in 1950s by Pierre-Paul Grasse during his research on termites. It is defined as a method of communication based on individuals modifying their surrounding environment.

${ }^{\mathrm{d}}$ The ACO is a bio-inspired (ants colony) probabilistic meta-heuristic for solving computational problems related to searching for an optimal path in a graph
} 
are available in the form of query-sessions (briefly sessions) that are formed by the query and by the different (possibly none) documents selected by the user among the results related to it; so, a session $s \in S$, where $S$ is the set of all the available sessions, is defined by

$$
s=(q, c),
$$

where $q \in Q$ is the query and $c=\left(d_{1}, \ldots, d_{|c|}\right) \in D_{q}^{|c|}$ is the ordered sequence of $|c|$ results selected by the user. Moreover, $D_{q} \subseteq D$ is the set of relevant documents (selected by the search engine) in relation to the query $q, Q$ being the set of all the known queries and $D$ the one of all the available documents.

Given a query $q \in Q$ and a document $d \in D_{q}$, the pheromone $w \in \mathbb{R}^{+}$associated to the couple $(q, d)$ is denoted by $\phi_{W}(q, d)$, where the function $\phi$ is defined by

$$
\phi: Q \rightarrow D \rightarrow \mathbb{R}^{+} \times T,
$$

while the last time the document was clicked among the results of the query is denoted by $\phi_{T}(q, d) \in T$.

Every time a result $d \in D_{q}$ is picked among the results of query $q \in Q$, or - carrying on the similarity with ACO - the path $q \rightarrow d$ is covered by one user, a certain quantity of pheromone is deposited on it. The straightforward implementation of ACO's principles, also described by Gayo-Avello and Brenes in their paper [8], is to employ the simplest incrementing function ever, namely the successor. Thus, the upgrade is issued applying the rule

$$
\phi_{W}(q, d)=\phi_{W}(q, d)+1 .
$$

Evaporation is obtained by an exponential decay, using the rule

$$
\phi_{W}(q, d)=\phi_{W}(q, d)\left(\frac{1}{2}\right)^{\frac{t-\phi_{T}(q, d)}{\delta}} .
$$

where $t \in T$ is the current timestamp and $\delta \in \mathbb{R}^{+}$is the time required for the pheromone to half its value.

Pheromone evaporation will be performed periodically for each pair query-document, always before the upgrade; the frequency of upgrading is related to the users' perceived relevancy of considered documents: evaporation is a useful mechanism to forget registered behaviors, thus issuing it frequently causes the increase of new registered behaviours' importance.

Finally, we consider the quantity of pheromone deposited on each pair query-document and rank results based on it; thus

$$
R_{\phi(q)}=\left\{\left(d_{i}, d_{j}\right): d_{i}, d_{j} \in D_{q} \wedge \phi_{W}\left(q, d_{i}\right) \geqslant \phi_{W}\left(q, d_{j}\right)\right\}
$$

defines the actual documents' ranking: for all known query $q \in Q$, the results' rank will be the one given by the chain $\left(D_{q}, R_{\phi(q)}\right)$.

Summarising, the ranking algorithm based on ACO proposed in this section employs pheromone's traces for each pair query-document; the pheromone increases every time a user selects a page among the results related to a query and, also, vaporizes itself in time, being a simple mechanism to take into account the gradual loss of interest by 
users. By doing this, once a user performs a known query the search engine is able to present a new ranking based on the behavior shown by users with the same informational need, by exploiting the pheromone's traces.

Thus, it's important to establish whether it's really possible to improve search engines' performance by employing this new approach; therefore, we devised an experiment involving online participants.

In the following section we will present details about setups and results.

\section{Evaluation}

We devised an experiment to test the hypothesis that a search engine employing the new approach based on ACO provides a new ranking based on users' behaviors, and that this new ranking somehow improves the users' degree of satisfaction in performing a search.

In fact, given that a search can be considered satisfactory if it's successful, i.e. if the user can easily (and quickly) identify the content he was looking for, we divided the experiment involving 8 participants (for the most part students of the faculty), asking them to find some contents and describing them only the informational need they have to solve (trying, as much as possible, not to give clues about how to formulate the query).

Although we are aware of the reduced size of the sample, its characteristics result compliant with demographics of search engine users, whom are more likely to employ our system in future. However, as a preliminary evaluation 8 users are enough, in fact, according to Nielsen and Landauer [14] conducting a usability testing with a single user a third of the usability problems will be discovered; with five or more users a little more can be gained. Nevertheless, if the goal is to run a controlled experiment, from which a statistical analysis has to be performed, at least twice this number is necessary [5]. Evaluations of user's satisfaction carried out by other disciplinary communities can also offer a proper methodology [21].

For sake of simplicity, we chose an existing search engine, adjusting the default ranking and using one of the proposed algorithms to compute the new one; the choice fell on the MediaWiki ${ }^{\text {e }}$ search engine - made available by Wikimedia Foundation to search contents among the famous online encyclopedia Wikipedia.

Consequently, we devised a search engine that works over the Wikipedia's contents and uses MediaWiki to fetch them; besides, it records the users' behavior and exploits it to improve the provided results' ranking by using one of the proposed algorithms.

Moreover, given the small number of participants available and to further ease the analysis, we chose to ignore the evaporation mechanism, since there's no need to adapt to the shifting of users' interests, which we reasonably assumed static.

We proposed six informational needs to be solved (summarized in table 1) to the 8 participants, providing them with the devised search engine that, even if it doesn't display the same Wikipedia graphic, offers the same information about the proposed results (the page title and a brief snippet); besides, the users were only allowed to use the provided search engine, without any time limitation or being in any way controlled by the examiners.

e http://wWw.mediawiki.org/wiki/MediaWiki 
Table 1. The six tasks submitted to the participants of the online experiment; the title of the page satisfying the task and the brief informational need's description given to the participants are reported.

\begin{tabular}{l|l}
\hline $\begin{array}{l}\text { Californium } \\
\begin{array}{l}\text { The chemical element which takes its name } \\
\text { from one of the United States. }\end{array}\end{array}$ & $\begin{array}{l}\text { Colostrum } \\
\text { The first milk a mother produces after giv- } \\
\text { ing birth. }\end{array}$ \\
\hline $\begin{array}{l}\text { Saturn } \\
\text { The last planet of the Solar System which } \\
\text { can be seen by naked eye. }\end{array}$ & $\begin{array}{l}\text { Japan } \\
\text { The country with the highest life ex- } \\
\text { pectancy. }\end{array}$ \\
\hline $\begin{array}{l}\text { John Von Neumann } \\
\text { The first computer virus's theorist. }\end{array}$ & $\begin{array}{l}\text { Beagle } \\
\text { The breed of dog which shares its name } \\
\text { with the ship on which Darwin did his ex- } \\
\text { plorations. }\end{array}$ \\
\hline
\end{tabular}

The search engine, in addition to recording the users' behavior, shows the content of the fetched pages next to the proposed results; this way one can also record the actions carried out inside each page, allowing us to build the entire click graph. These information were included in the ranking computation too, i.e. considering not only the unit length's paths, but also the longer ones; thus we can operate also among those pages considered less relevant by MediaWiki, but considered relevant by the users, even if they reached them with a higher number of clicks than the ones displayed in the results.

\subsection{Results}

If the proposed framework somehow improves over the default ranking we should witness some changes in a way that could better satisfy new users facing the same informational needs than their predecessors; moreover - given that our approach closely follows the ACO approach's suggestions - in order for the system to exploit the collective behavior of its users, the queries submitted to the search engine shouldn't be too heterogeneous, thus even with such a limited number of users' interactions the system could still be effective. Naturally, given the uncontrolled and limited nature of the experiment, the results will be taken as preliminary and only have informative value, but we can still rely on them to better understand some underlying dynamics driving the users during a search, and to analyze how the algorithm performs in a real confined environment.

Once the experiment ended, we gathered th $\square$ ry-click logs produced and analyzed them in order to extract the evolution of the 1 none deposited of the key pages solving each task; the charts depicted in figure 4.1 summarise the results of our experiment: each figure reports the findings for one single task and shows on the $\mathrm{x}$-axis a timeline of the interactions occurred between the participants and our system. On the y-axis the quantity of pheromone deposited on the document solving that particular task is shown, related to the different queries issued by the users shown in colors. Every successful interaction corresponds to one point on the graph; the color represent the ranking status in which the interaction occurred: the circle one means that the page wasn't found on the first result for the query issued by the user, thus being a disadvantageous situation for users which has to seek for the right page among the other results; whereas the 
star shape indicates that the page was the first appearing among the results, thus being an advantageous situation for users which could immediately spot the answer for their informational need among the list of results.
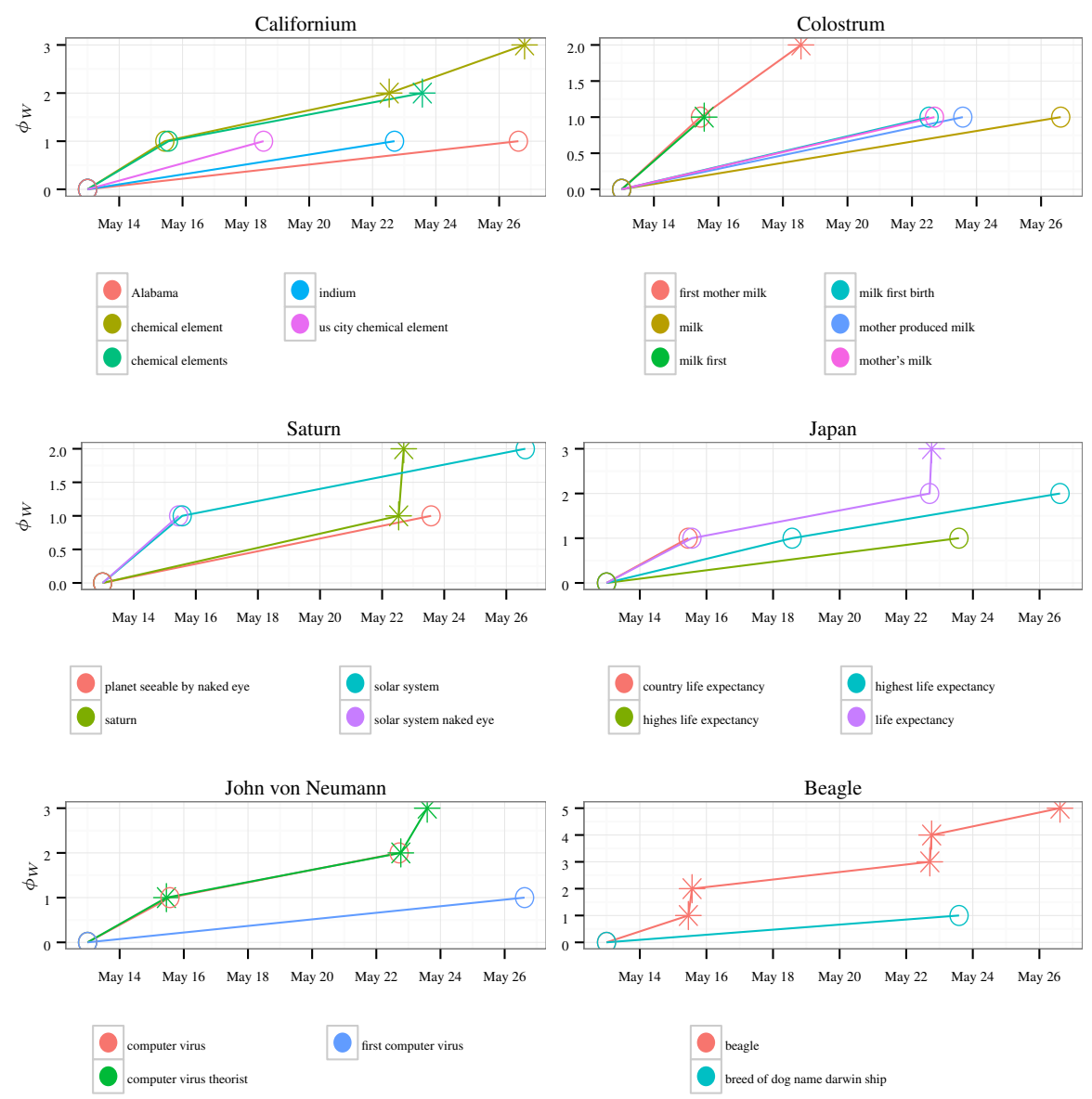

$$
\text { Page ranking } \bigcirc_{i 1} \text { 米 } 1
$$

Fig. 1. Results obtained from the online experiment; the stars indicate that the page solving the task became the first in the ranking.

In general the tasks were solved by the majority of participants, since all of them were solved by at least 6 out of 8 participants, with the "Japan" task solved by 7 and the "Californium" one by everyone; this variation may be caused by several factors, e.g. difficulties encountered by users in performing effective queries to solve specific tasks, such as the "Beagle" one, which was apparently solved indirectly by 5 out of 6 participants, who then searched for the right page. 
As we expected, queries performed by a few users - even though they managed to solve the task - don't really affect the ranking in favor of the goal page; indeed users solving tasks through non-specific queries need a higher number of clicks, given that the results aren't enough specific and thus a more accurate analysis. This way, some pheromone has been deposited even onto documents not solving the particular user's informational need, thus voiding the effect of finally reaching the right page among the results.

Furthermore, another beneficial effect noticed through the experiment to be pointed out is the stability yielded by the optimal ranking, since the goal page keeps appearing in the first position of the ranking once earned; this is caused by the algorithm's underlying approach employing user's behaviors, but could also reveal some degree of self-reinforcement in the ranking algorithm: in fact, when a page reaches the first position among the results, it will be prone to be selected by the majority of users, causing the progressive increase of its pheromone and a convergence to a suboptimal solution. Although in our case the domain makes this effect negligible (it's unlikely that the computed ranking will stop being the optimal one, since the information contained into an encyclopedia is rather static), in reality here is when the evaporation mechanism takes place, making the ranking more flexible thus preventing the system's convergence to a non-optimal solution.

In conclusion, from our results we could argue that our algorithm could offer a considerable improveme $\bigcirc$ the online search experience, in the case of informational queries; thus, it could . . .elpful to consider further relevance measures in our ranking computation, such as LAR-based ones.

\section{Conclusion}

Recently, Google introduced their Social Search service declaring: "with these changes, we want to help you finding the most relevant information from the people who matter to you". That is, in a way, our definition of a colony. The mechanism is the Google+1 buttor $\square$ ich let users share interesting pages with their contacts - a way of releasing pherc 7 :

These ease show the paradigm shift in Web searching that we are experiencing today. We hope that our approach can be a first step in modeling and describing these trends.

We designed an algorithm employing an ACO strategy to provide implicit collaborative seeking features in real-time to search engines. It seems particularly adequate for informational queries for retrieving results about relatively static information on the Web, such as: looking for products in a catalogue or encyclopedia entries.

We evaluated the algorithm by designing an online experiment.

Since our methods are based on users interactions to discover the most promising results, the datasets were partitioned into a training set and a test set. The online evaluation consisted of an experiment with 8 participants employing the MediaWiki search engine augmented with our NaïveRank algorithm. It proved to be effective for the sample set of participants and was relevant to get a first insight about our approach with real users. In future we plan to extend the online experiment to a more extended sample of participants and test the other two algorithms in a real-time scenario with users. We hope to prove that also in an online environment real-time relevant results can be obtained by 
users employing an implicit collaborative approach for information seeking and selecting the right algorithm depending in the types of queries.

\section{References}

1. Broder, A.: A taxonomy of web search. ACM Sigir forum 36(2), 3-10 (Sep 2002), http: //portal.acm.org/citation.cfm?doid=792550.792552

2. Calhoun, K.: The changing nature of the catalog and its integration with other discovery tools (2006), http://www.loc.gov/catdir/calhoun-report-final.pdf

3. Dempsey, L.: Thirteen ways of looking at libraries, discovery and the catalogue: scale, workflow, attention (2013), http://scholar.google.com/scholar?q=related: nvQM9zcUrdc J: scholar.google.com/\&hl=en\&num=20\&as_sdt =0, 5

4. Devine, J., Egger-Sider, F.: Going beyond Google again (2014), http: / / cds . cern . ch / record/1735423

5. Dix, A.: Human-computer interaction: A stable discipline, a nascent science, and the growth of the long tail. Interacting with Computers 22(1), 13-27 (Jan 2010), http://iwc . oxfordjournals.org/cgi/doi/10.1016/j.intcom.2009.11.007

6. Exner, N.: Research Information Literacy: Addressing Original Researchers' Needs. The Journal of Academic Librarianship 40(5), 460-466 (Sep 2014), http: / / linkinghub . elsevier.com/retrieve/pii/s0099133314000901

7. Fox, S., Karnawat, K., Mydland, M., Dumais, S., White, T.: Evaluating implicit measures to improve web search. Acm Transactions on Information Systems 23(2), 147-168 (Apr 2005), http://portal.acm.org/citation.cfm?doid=1059981.1059982

8. Gayo-Avello, D., Brenes, D.J.: Making the road by searching - A search engine based on Swarm Information Foraging. arXiv.org (Nov 2009), http: / / arxiv . org/abs / 0911. 3979v1

9. Goldberg, D., Nichols, D., Oki, B.M., Terry, D.: Using Collaborative Filtering to Weave an Information Tapestry. Communications of the ACM 35(12), 61-70 (Dec 1992), http: //portal.acm.org/citation.cfm?doid=138859.138867

10. Hawking, D., Craswell, N., Bailey, P., Griffihs, K.: Measuring search engine quality. Information Retrieval 4(1), 33-59 (2001), http: //link.springer.com/10.1023/A: 1011468107287

11. Hoeppner, A.: The ins and outs of evaluating web-scale discovery services: Librarians around the world are trying to learn what WSD services are and how they work. Computers in Libraries (2012), http://www.questia.com/magazine/1G1-286254697/theins-and-outs-of-evaluating-web-scale-discovery

12. Hull, D., Pettifer, S.R., Kell, D.B.: Defrosting the digital library: bibliographic tools for the next generation web. PLoS computational biology (2008), http://dx.plos.org/10. 1371/journal.pcbi.1000204.g005

13. Lawrence, S., Giles, C.L.: Accessibility of information on the Web. intelligence 11(1), 32-39 (Apr 2000), http: / / dl.acm.org/ft_gateway. cfm?id=333181\&type=html

14. Nielsen, J., Landauer, T.K.: A mathematical model of the finding of usability problems. In: CHI '93: Proceedings of the INTERACT '93 and CHI '93 Conference on Human Factors in Computing Systems. pp. 206-213. ACM Request Permissions, New York, New York, USA (May 1993), http://portal.acm.org/citation.cfm?doid= 169059.169166

15. Olston, C., Chi, E.H.: ScentTrails: Integrating browsing and searching on the Web. Transactions on Computer-Human Interaction (TOCHI 10(3), 177-197 (Sep 2003), http: // portal.acm.org/citation.cfm?doid=937549.937550

16. Quint, B.: Attacking Our Problems. Information Today 31(2), 8 (Mar 2014) 
17. Richardson, H.: Revelations from the Literature: How Web-Scale Discovery Has Already Changed Us. Computers in Libraries (2013), http://eric.ed.gov/?id= EJ1014245

18. Rose, D.E., Levinson, D.: Understanding user goals in web search. In: WWW '04: Proceedings of the 13th international conference on World Wide Web. pp. 13-19. ACM, New York, New York, USA (May 2004), http://portal.acm.org/citation. cfm?doid= 988672.988675

19. Silverstein, C., Marais, H., Henzinger, M., Moricz, M.: Analysis of a very large web search engine query log. SIGIR Forum 33(1), 6-12 (Sep 1999), http: / / portal . acm.org/ citation.cfm?doid=331403.331405

20. Thomsett-Scott, B., Reese, P.E.: Academic Libraries and Discovery Tools: A Survey of the Literature. dx.doi.org 19(2-4), 123-143 (Aug 2012), http: / / www . tandfonline. $\mathrm{com} / \mathrm{doi} / \mathrm{abs} / 10.1080 / 10691316.2012 .697009$

21. Vaughan, J.: Web Scale Discovery Services (2011), http://books.google.co.uk/ books/about/Web_Scale_Discovery_Services.html? id=d74 J5 nN9MmMC

22. Wiener, N.: The Human Use of Human Beings: Cybernetics and Society. A Da Capo paperback, Da Capo Press (1954), http://www.worldcat.org/title/human-useof-human-beings-cybernetics-and-society/oclc/17353835

23. Wu, J., Aberer, K.: Swarm intelligent surfing in the Web. Human-Computer Interaction - Interact 2005, Proceedings 2722, 431-440 (2003), http://gateway. webofknowledge. com/gateway/Gateway. cgi?GWVersion=2\&SrcAuth= mekentos j\&SrcApp=Papers\&Dest LinkType=Full Record\&DestApp=WOS\& KeyUT $=000185074000080$

24. Xie, Y., O'Hallaron, D.: Locality in search engine queries and its implications for caching. In: INFOCOM 2002. Twenty-First Annual Joint Conference of the IEEE Computer and Communications Societies. Proceedings. IEEE. pp. 1238-1247. IEEE (2002), http://ieeexplore.ieee.org/lpdocs/epic03/wrapper.htm? arnumber $=1019374$

25. Xu, Y., Mease, D.: Evaluating web search using task completion time. In: SIGIR '09: Proceedings of the 32nd international ACM SIGIR conference on Research and development in information retrieval. pp. 676-677. ACM, New York, New York, USA (Jul 2009), http://portal.acm.org/citation.cfm?doid=1571941.1572073

26. Young, M., Yu, H.: The impact of web search engines on subject searching in OPAC. Information technology and libraries 23(4), 168-180 (2004) 